\title{
$\begin{array}{llllll}\text { January } & 28, & 2016 & 12: 15 & \text { WSPC/INSTRUCTION } & \text { FILE }\end{array}$
}

\section{Self-alignment driven by jump processes: macroscopic limit and numerical investigation}

\author{
Giacomo Dimarco \\ Department of Mathematics and Computer Science; \\ University of Ferrara; \\ Ferrara, Italy \\ giacomo.dimarco@unife.it \\ Sebastien Motsch \\ School of Mathematical \& Statistical Sciences; \\ Arizona State University; \\ Tempe, Arizona USA. \\ email: smotsch@asu.edu \\ Received (Day Month Year) \\ Revised (Day Month Year) \\ Communicated by (xxxxxxxxxx)
}

\begin{abstract}
In this paper, we are interested in studying self-alignment mechanisms described as jump processes. In the dynamics proposed, active particles are moving at a constant speed and align with their neighbors at random times following a Poisson process. This dynamics can be viewed as an asynchronous version of the so-called Vicsek model. Starting from this particle dynamics, we introduce the related kinetic description and then derive a continuum hydrodynamic model. We then introduce different discretization strategies for the hierarchy of proposed models, we numerically study the convergence of the schemes and compare the behaviors of the different systems for several test cases.
\end{abstract}

Keywords: Relaxation system, macroscopic limit, Vicsek model, self-organized hydrodynamic, self-propelled particles, semi-Lagrangian schemes.

AMS Subject Classification: 35Q92, 35L40, 35L67, 65M12, 76M12, 82B80

\section{Introduction}

We are interested in the mathematical modeling and numerical simulation of collective motion in systems composed by a large number of agents. Typical examples are flock of birds, fish schools, suspensions of active swimmers such as bacteria or cells. This field of research is nowadays an extremely active topic both in the mathematical community ${ }^{6,7,17,36}$ as well as in the physics community ${ }^{43,32}$. The problem of describing this kind of motion can be and has been historically tackled from different points of view and with different levels of detail. The first and more natural approach consists in describing these systems by introducing laws that each individual 


$\begin{array}{lllll}\text { January } & 28, & 2016 & 12: 15 & \text { WSPC/INSTRUCTION }\end{array}$

should follow. We refer to this approach as particle model or Individual Based Model (IBM) $9,10,11,13,28,30,33,35$. At the next level of detail, we find the so-called kinetic approaches which describe the time evolution of such microscopic systems using a probabilistic viewpoint through a density distribution. In this description, a given agent or particle does not have a definite position or velocity but instead certain probabilities of having different positions and velocities. We refer to [36] for a recent review on this kind of approach. Finally, one can describe large systems of interacting particles by means of macroscopic models, in this case only the evolution of local average quantities such as the density and average velocity of the particles are typically studied ${ }^{32}$. The study of the transition from one model to the other is crucial to better understand the possible use and range of validity of each description. The derivation of macroscopic models from microscopic ones has been intensively studied theoretically by many authors in the recent past ${ }^{4,5,8,19,23,26,38,40,41}$. However, a similar analysis have not been yet performed with the same accuracy from a numerical standpoint. Only few numerical comparisons between models have been investigated in the context of self-organized dynamics ${ }^{1,34}$. Thus, there is a need for a more systematic comparisons between models and numerical techniques to determine in which regimes (e.g. number of individual, range of parameters) each description works best. This work represents an attempt in this direction.

In this paper, we consider at the microscopic level (i.e. IBM) an interacting particle system which self-align due to a biased jump process. In this dynamics, agents move at a constant speed while their direction of motion are randomly updated towards the average velocity of their neighbors. One can view this dynamics as an asynchronous version of the Vicsek model ${ }^{42}$. Even though the primary goal of this study is to investigate alignment interaction, it is clear that this dynamics can be generalized to take into account other type of interaction such as attraction and repulsion ${ }^{3,12}$. Many aspects of the Vicsek model have been extensively studied such as phase transitions ${ }^{2,10,16,27,42}$, numerical simulations ${ }^{18,34,42}$, derivation of macroscopic models ${ }^{8,14,15,19}$. We perform similar studies for the model proposed.

The first goal is to derive a macroscopic system of equations, a so-called selforganized hydrodynamic ( $\mathrm{SOH}$ ), starting from the proposed microscopic jump process. In order to derive the corresponding macroscopic model, we use an intermediate kinetic description which studies the evolution of the one-particle distribution in phase space. Here, the kinetic description of the jump process is given by a Bhatnagar-Gross-Krook (BGK) operator. The second goal is to perform a numerical study and comparison of the three models introduced: the microscopic, the mesoscopic and the macroscopic one. With this aim, we introduce ad hoc discretizations of the systems and compare them trying to highlight the different behaviors expected from such models.

In many fields of research where numerical simulations play a fundamental role (e.g. fluid-dynamics, plasma physics or structural engineering), there exists a number of benchmark tests which permit to evaluate the efficiency and the performances of new numerical methods. In these fields, the validity of the mathematical or phys- 
ical models are well established and only the numerical schemes have to be tested. In biology, numerical simulations are also used to assess the pertinence of the model in describing various phenomena. However, there is a need to develop 'standardized tests' to examine and compare different models, in the same vein as physicists aim at characterizing phase transitions ${ }^{10,42}$. It will be suitable that once that a new model is proposed which shares similarities with pre-existing dynamics, we could use a battery of tests to examine whether a models give similar results. For instance, in fish modeling, a systematic comparison between several dynamics has been performed in [37] leading to a 'meta-analysis'. This paper advocates in starting to systematize such approach by introducing some simple tests which (i) assess the validity of the numerical methods by performing a numerical convergence analysis, (ii) identify typical features of the models proposed by running several Riemann problems and smooth solutions (Vortex configuration) and (iii) compare the different scale dynamics: microscopic, mesoscopic and macroscopic one. With this idea in mind, we plan to perform in a near future a study aiming at comparing several models of active particles already present in literature.

The outline of the paper is as follows. In section 2, we introduce the model hierarchy and the main results. It consists of the IBM, the kinetic and the hydrodynamic limits. In Section 3 we will detail the numerical schemes used to discretize the different models. In Section 4 we introduce the benchmark tests and we analyze the different solutions obtained. Section 5 is devoted to draw a conclusion and to open new perspectives.

\section{The model}

In this section we introduce a hierarchy of models describing self-alignment through jump process at various scales. We first start from a system of interacting particles (e.g. IBM model), then we introduce the associated kinetic description and finally we derive the hydrodynamic limit of such dynamics.

\subsection{Individual-Based Model}

We consider a system of $N$-particles moving in $\mathbb{R}^{d}$, where $d$ is the spatial dimension $(d=2$ or 3$)$. Each particle $k \in\{1, \ldots, N\}$ is described by its position $\mathbf{x}_{k}(t) \in \mathbb{R}^{d}$ and its orientation $\omega_{k}(t) \in \mathbb{S}^{d-1}$ :

$$
\dot{\mathbf{x}}_{k}=c \omega_{k},
$$

where $c$ is the (constant) speed of the particle. The evolution of the orientation $\omega_{k}(t)$ is modeled as a jump process where the jumps occur according to a Poisson process with a constant rate $\nu>0$. Thus, the trajectory of the particle $\mathbf{x}_{k}$ is piece-wise linear (see figure 1). The new direction of motion $\omega_{k}^{*}$ is sampled from the following density distribution:

$$
P\left(\omega_{k} \rightarrow \omega_{k}^{*}\right)=\phi\left(\Omega_{k} \cdot \omega_{k}^{*}\right) .
$$


Here, $\phi$ is a given density distribution that satisfies:

$$
\int_{\omega \in \mathbb{S}^{d-1}} \phi(\Omega \cdot \omega) \mathrm{d} \omega=1, \quad \text { for any } \Omega \in \mathbb{S}^{d-1} .
$$

The unit vector $\Omega_{k}$ is the average direction at $\mathbf{x}_{k}$ (see figure 1 ). To estimate this average, we compute the local flux $\mathcal{J}\left(\mathbf{x}_{k}\right)$ which sums up the velocities direction $\omega_{i}$ in the zone of influence of $\mathbf{x}_{k}$ and then we normalize this local flux:

$$
\Omega_{k}=\frac{\mathcal{J}\left(\mathbf{x}_{k}\right)}{\left|\mathcal{J}\left(\mathbf{x}_{k}\right)\right|}, \quad \mathcal{J}\left(\mathbf{x}_{k}\right)=\frac{1}{N} \sum_{i=1}^{N} K\left(\left|\mathbf{x}_{k}-\mathbf{x}_{i}\right|\right) \omega_{i}
$$

The zone of influence is encoded in the influence kernel $K(|\mathbf{x}|)$ which is supposed smooth at the origin and compactly supported (e.g. an indicator function). Notice that the new velocity $\omega_{k}^{*}$ is independent of $\omega_{k}$.

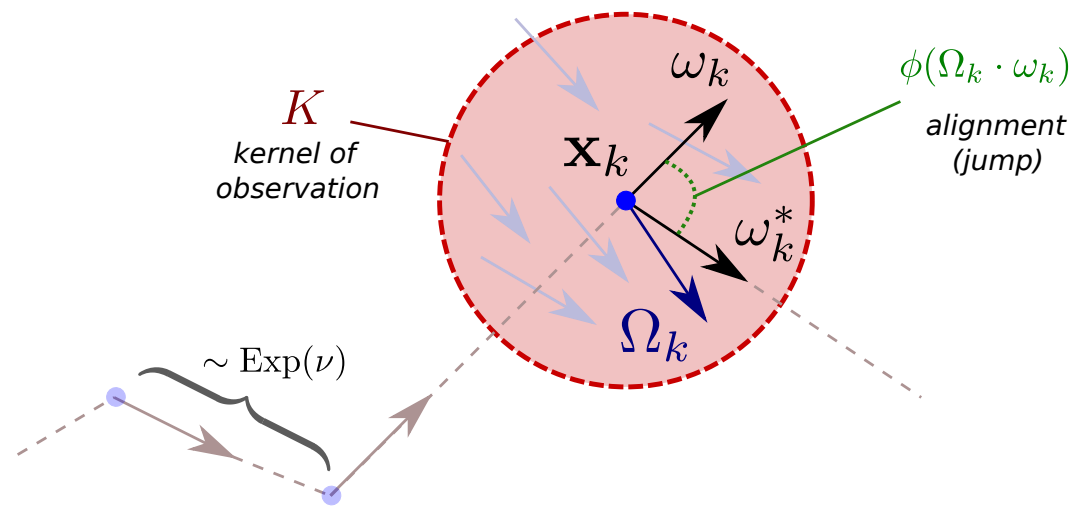

Fig. 1. Illustration of the velocity jump process. The particle $k$ changes its direction $\omega_{k}$ according to a Poisson process with rate $\nu$. Once a jump occurs, the new direction $\omega_{k}^{*}$ is sample from the distribution $\phi$ centered around $\Omega_{k}$ the average direction of the local neighbors (represented in red).

Remark 2.1. The evolution of $\omega_{k}$ is similar to a compound Poisson Process ${ }^{22}$ :

$$
\omega_{k}(t)=\Delta \omega_{1}+\cdots+\Delta \omega_{N(t)}
$$

where $N(t)$ is a Poisson process with intensity $\nu$ and $\Delta \omega_{j}$ are random variables with laws given by $\Delta \omega_{j}=\omega_{k}^{*}-\omega_{k}$ where $\omega_{k}^{*}$ is distributed according to (2.2).

Since the time of occurrence of jumps for $\omega_{k}$ is modeled as a Poisson process, the probability that a jump occurs in a time interval $\Delta t$ is given by:

$$
\operatorname{Prob}\left(\omega_{k} \text { "jumps during" }[t, t+\Delta t]\right)=1-\mathrm{e}^{-\nu \Delta t} .
$$

If no jump occurs, the particle $k$ keeps moving straight during the time interval $[t, t+\Delta t]$. 
Several choices of distribution $\phi$ can be made. For instance, in the original Vicsek model ${ }^{42}$, as the dynamics takes place in a plane, velocities $\mathbf{v}_{k}$ are solely described using angle direction $\theta_{k}^{*}$. The post-jump angle direction of a particle is given by:

$$
\theta_{k}^{*}=\theta_{\Omega_{k}}+\varepsilon,
$$

where $\theta_{\Omega_{k}}$ denotes the angle direction of the average direction $\Omega_{k}$ and $\varepsilon$ is a random variable with uniform law on the interval $[-\eta, \eta]$. Thus, $\eta$ indicates the level of 'noise' in the dynamics (i.e. the larger $\eta$ is, the more 'noisy' is the dynamics). To have a similar dynamics in our framework, we have to take:

$$
\phi(s)=\left\{\begin{array}{l}
C \text { if }|s| \leq \arccos (\eta) \\
0 \text { otherwise }
\end{array}\right.
$$

where $C$ is a normalization constant. With this choice for $\phi$, we obtain the same law for the jump velocity as in the original Vicsek model. The only difference is that in our dynamics the jumps do not occur all at the same time but are distributed in time according to a Poisson process. For this reason, we refer to the dynamics (2.1)(2.2) with $\phi$ given by (2.5) as the asynchronous Vicsek model.

Another choice for $\phi$ is given by a Von Mises distribution:

$$
\phi(\cos \theta)=C \exp \left(\frac{\cos \theta}{\sigma}\right),
$$

where $\sigma$ denotes the spread of the distribution corresponding to the level of 'noise' and $C$ is once again a normalization constant. The angle $\theta$ is the angle between the average orientation of the particles $\Omega$ and the actual orientation of the particle. The Von Mises distributions correspond to equilibria of the dynamics introduced in [19]. We will show in the next section that the velocity jump process $(2.1)(2.2)$ will also provide Von Mises distributions as equilibrium. However, as it will be clear in the next section, the average orientation $\Omega$ will differ between the two systems (the one presented here and the one presented in [20]) leading to different asymptotic dynamics.

\subsection{The kinetic model}

\subsubsection{Empirical distribution}

Starting from the velocity jump process described in the previous paragraph, we are interested in finding the mesoscopic description associated with this dynamics. Let $f(\mathbf{x}, \omega, t)$ be the density of particles at time $t \geq 0$ with position $\mathbf{x} \in \mathbb{R}^{d}$ and orientation $\omega \in \mathbb{S}^{d-1}$. As the number of particles $N$ goes to infinity, we obtain formally that the distribution $f$ satisfies the following master equation:

$\partial_{t} f+c \omega \cdot \nabla_{\mathbf{x}} f=\nu\left(\int_{\widetilde{\omega} \in \mathbb{S}^{d-1}} P(\widetilde{\omega} \rightarrow \omega) f(\mathbf{x}, \widetilde{\omega}) \mathrm{d} \widetilde{\omega}-\int_{\omega^{*} \in \mathbb{S}^{d-1}} P\left(\omega \rightarrow \omega^{*}\right) f(\mathbf{x}, \omega) \mathrm{d} \omega^{*}\right)$,

where the right-hand side counts the number of particles entering and leaving $(\mathbf{x}, \omega)$. The rigorous derivation of such equation is out of the scope of the paper, it requires 
to prove the so-called propagation of $\operatorname{chaos}^{29,31,39}$ which appears not to be straightforward. Using the expression of the transition probability $P\left(\omega \rightarrow \omega^{\prime}\right)(2.2)$, we obtain the kinetic equation:

$$
\partial_{t} f+c \omega \cdot \nabla_{\mathbf{x}} f=\nu\left(\rho \phi_{\Omega_{f}}-f\right) .
$$

Here, $\nu>0$ is the jump frequency while $\rho(\mathbf{x}, t)$ is the density of particles at the position $\mathbf{x}$ and time $t$ :

$$
\rho(\mathbf{x}, t)=\int_{\omega \in \mathbb{S}^{d-1}} f(\mathbf{x}, \omega, t) \mathrm{d} \omega .
$$

The distribution $\phi_{\Omega_{f}}$ describes the post-jump distribution velocity:

$$
\phi_{\Omega_{f}}(\omega)=\phi\left(\Omega_{f} \cdot \omega\right),
$$

where the mean direction $\Omega_{f}$ is defined in a similar way as in the Individual-Based model (2.4), we simply have to change a sum into an integral:

$$
\Omega_{f}(\mathbf{x}, t)=\frac{\mathcal{J}_{f}(\mathbf{x}, t)}{\left|\mathcal{J}_{f}(\mathbf{x}, t)\right|}, \quad \mathcal{J}_{f}(\mathbf{x}, t)=\int_{\mathbf{y} \in \mathbb{R}^{d}} \int_{\omega \in \mathbb{S}^{d-1}} K(|\mathbf{y}-\mathbf{x}|) f(\mathbf{y}, \omega, t) \omega \mathrm{d} \mathbf{y} \mathrm{d} \omega .
$$

The operator $K$ denotes as before the influence kernel.

Remark 2.2. Notice that the collisional operator given by the right-hand-side of (2.7) is a BGK-type operator (i.e. relaxation of $f$ toward a given distribution $\rho \phi_{\Omega_{f}}$ ). In contrast to the Fokker-Planck operator in [19], this operator preserves the mean direction $\Omega_{f}(\mathbf{x})$. In other words, in the homogeneous case in space (i.e. $f$ independent of $\mathbf{x}$ ), the mean direction $\Omega_{f}$ would remain constant over the relaxation to equilibrium time while this was not the case for the Fokker-Planck equation in [19].

BGK type operators are generally obtained as an approximation of Boltzmann equations? . Here, there is no such approximation since the particles in the microscopic model are supposed to follow a jump process. If the particles would follow a Boltzmann type equation ${ }^{25}$, then we would have to make such approximation to obtain a BGK equation at the kinetic level.

\subsubsection{Dimensionless variables}

In order to highlight the main features of the above model, we can introduce the following dimensionless variables:

$$
\tilde{t}=t / t_{0} \quad, \quad \tilde{\mathbf{x}}=\mathbf{x} / \mathbf{x}_{0},
$$

where $t_{0}$ and $\mathbf{x}_{0}$ are characteristic time and length. Choosing $t_{0}=\nu$ and $\mathbf{x}_{0}=c / \nu$ and writing $f$ in these new variables $\tilde{f}(\tilde{\mathbf{x}}, \omega, \tilde{t}) \mathrm{d} \tilde{\mathbf{x}}=f(\mathbf{x}, \omega, t) \mathrm{d} \mathbf{x}$, the kinetic equation reduces to:

$$
\partial_{t} f+\omega \cdot \nabla_{\mathbf{x}} f=\rho \phi_{\Omega_{f}}-f
$$


where the density $\rho$ and distribution $\phi_{\Omega_{f}}$ are defined in (2.8) and (2.10) respectively. The kernel of observation $K$ has also to be written in dimensionless variables: $\tilde{K}(\tilde{\mathbf{x}})=K(\mathbf{x})$.

\subsubsection{Micro-macro scales}

We are now interested in studying regimes where the effects of interactions between agents are strong. With this aim, we rescale space and time variables by introducing the ratio $\varepsilon$ between the microscopic and macroscopic scales

$$
t^{\prime}=\varepsilon t \quad, \quad \mathbf{x}^{\prime}=\varepsilon \mathbf{x} .
$$

When $\varepsilon \ll 1$, interactions becomes more important meaning that alignment acts at a high frequency but the interaction becomes also very localized in space. We write down the distribution of particle in these new variables: $f^{\varepsilon}\left(\mathbf{x}^{\prime}, \omega, t^{\prime}\right)=\frac{1}{\varepsilon^{d}} f(\mathbf{x}, \omega, t)$. The evolution of $f^{\varepsilon}$ is governed by the following equation

$$
\partial_{t^{\prime}} f^{\varepsilon}+\omega \cdot \nabla_{\mathbf{x}^{\prime}} f^{\varepsilon}=\frac{1}{\varepsilon}\left(\rho^{\varepsilon} \phi_{\Omega_{f^{\varepsilon}}}-f^{\varepsilon}\right),
$$

where $\rho^{\varepsilon}$ denotes the spatial distribution:

$$
\rho^{\varepsilon}\left(\mathbf{x}^{\prime}, t^{\prime}\right)=\int_{\omega \in \mathbb{S}^{d-1}} f^{\varepsilon}\left(\mathbf{x}^{\prime}, \omega, t\right) \mathrm{d} \omega
$$

and $\Omega_{f}$ is the average direction in macroscopic variables:

$\Omega_{f^{\varepsilon}}\left(\mathbf{x}^{\prime}, t^{\prime}\right)=\frac{\mathcal{J}^{\varepsilon}\left(\mathbf{x}^{\prime}, t^{\prime}\right)}{\left|\mathcal{J}^{\varepsilon}\left(\mathbf{x}^{\prime}, t^{\prime}\right)\right|}, \mathcal{J}^{\varepsilon}\left(\mathbf{x}^{\prime}, t^{\prime}\right)=\int_{\left(\mathbf{y}^{\prime}, \omega\right) \in \mathbb{R}^{d} \times \mathbb{S}^{d-1}} K\left(\frac{\left|\mathbf{y}^{\prime}-\mathbf{x}^{\prime}\right|}{\varepsilon}\right) f^{\varepsilon}\left(\mathbf{y}^{\prime}, \omega, t^{\prime}\right) \omega \mathrm{d} \mathbf{y}^{\prime} \mathrm{d} \omega$.

In the following we drop the primes for clarity. Now notice that the average direction $\Omega_{f}^{\varepsilon}$ becomes local in space as $\varepsilon \rightarrow 0$. More precisely, by a change of variables and Taylor expansion, we have

$$
\Omega_{f^{\varepsilon}}(\mathbf{x}, t)=\bar{\Omega}_{f^{\varepsilon}}(\mathbf{x}, t)+O\left(\varepsilon^{2}\right),
$$

with

$$
\bar{\Omega}_{f^{\varepsilon}}(\mathbf{x}, t)=\frac{j_{f^{\varepsilon}}(\mathbf{x}, t)}{\left|j_{f^{\varepsilon}}(\mathbf{x}, t)\right|}, \quad j_{f^{\varepsilon}}(\mathbf{x}, t)=\int_{\omega \in \mathbb{S}^{d-1}} f^{\varepsilon}(\mathbf{x}, \omega, t) \omega \mathrm{d} \omega .
$$

Thus, supposing the function $\phi$ is smooth, we deduce the following expression for the equation satisfied by $f^{\varepsilon}$

$$
\partial_{t} f^{\varepsilon}+\omega \cdot \nabla_{\mathbf{x}} f^{\varepsilon}=\frac{1}{\varepsilon} Q\left(f^{\varepsilon}\right)+O(\varepsilon)
$$

with $Q(f)$ the relaxation operator defined as:

$$
Q(f)(\mathbf{x}, \omega)=\rho(\mathbf{x}) \phi_{\bar{\Omega}_{f}}(\omega)-f(\mathbf{x}, \omega),
$$

where $\rho(\mathbf{x})$ and $\bar{\Omega}_{f}(\mathbf{x})$ are defined in (2.8) and (2.14). In the following, we will omit the superscript $\varepsilon$ if not strictly necessary for clarity. 


\subsection{The macroscopic model}

In this section we are interested in exploring the formal limit $\varepsilon \rightarrow 0$. We refer to it to as the hydrodynamic limit. To investigate such a limit, we first study the properties of the operator $Q(2.15)$. With this aim, we are first interested in the homogeneous case in space

$$
Q(f)(\omega)=\rho \phi_{\bar{\Omega}_{f}}(\omega)-f(\omega)
$$

with $\rho$ and $\bar{\Omega}_{f}$ given by:

$$
\rho=\int_{\omega \in \mathbb{S}^{d-1}} f(\omega) \mathrm{d} \omega, \quad \bar{\Omega}_{f}=\frac{j_{f}}{\left|j_{f}\right|} \quad \text { with } j_{f}=\int_{\omega \in \mathbb{S}^{d-1}} f(\omega) \omega \mathrm{d} \omega .
$$

Since the operator $Q$ is a relaxation operator, the equilibrium functions satisfying $Q(f)=0$ are given by

$$
f(\omega)=\rho \phi_{\bar{\Omega}_{f}}(\omega) .
$$

Thus, equilibria are fully described by the two macroscopic quantities $\rho$ and $\bar{\Omega}_{f}$. To find the time evolution of those quantities, we investigate the moments of $f$. In order to find a closed form for the moments equations we need to find a complete set of collisional invariants of $Q$ if they exist. In this context, a function $\psi(\omega)$ is called a collisional invariant if it satisfies:

$$
\int_{\omega \in \mathbb{S}^{d-1}} Q(f) \psi(\omega) \mathrm{d} \omega=0, \quad \text { for any } f(\omega) .
$$

Now, it is clear that the constant function $\psi=1$ is a collisional invariant since:

$$
\int_{\omega \in \mathbb{S}^{d-1}} Q(f) \mathrm{d} \omega=0
$$

This means that the operator $Q$ preserves the total mass of particles. However, the constant 1 spans only a one-dimensional function space, while the set of equilibria is a $d$-dimensional manifold (where $d$ is the spatial dimension). Thus in order to close the system we need to find other invariants. Unfortunately, in contrast to classical physics, the operator $Q$ does not preserve any other quantities. Indeed, for any test function $\psi$ satisfying $\int_{\omega \in \mathbb{S}^{d-1}} \psi(\omega) 1 \mathrm{~d} \omega=0$, we have

$$
\int_{\omega \in \mathbb{S}^{d-1}} Q(\psi) \psi \mathrm{d} \omega=\int_{\omega \in \mathbb{S}^{d-1}}-\psi^{2} \mathrm{~d} \omega .
$$

Thus, $\psi$ cannot be a (non-trivial) collisional invariant. To overcome the lack of collisional invariants, we introduce a weak notion of invariants, a so-called class of generalized collisional invariants (GCI), following the idea introduced in [19].

Definition 2.1. Fix a direction $\Omega_{*}$. A function $\psi_{\Omega_{*}}$ is a Generalized Collisional Invariant (GCI) of the operator $Q$ associated with the vector $\Omega_{*}$ if it satisfies:

$$
\int_{\omega \in \mathbb{S}^{d-1}} Q(f) \psi_{\Omega_{*}}(\omega) \mathrm{d} \omega=0,
$$


for any $f$ such that $\bar{\Omega}_{f}$ given by $(2.16)$ is proportional to $\Omega_{*}$. In other words, $f$ satisfies the constraint:

$$
P_{\Omega_{*}^{\perp}}\left(\int_{\omega \in \mathbb{S}^{d-1}} f(\omega) \omega \mathrm{d} \omega\right)=0,
$$

with $P_{\Omega_{*}^{\perp}}=\mathrm{Id}-\Omega_{*} \otimes \Omega_{*}$ orthogonal projection to $\Omega_{*}$

Thus, in the definition of GCI, we reduce the domain of the test functions $\psi$. To find the GCI, we notice that for any $\Omega_{*} \in \mathbb{S}^{d-1}$ we have

$$
\int_{\omega \in \mathbb{S}^{d-1}} \phi\left(\Omega_{*} \cdot \omega\right) \omega \mathrm{d} \omega=c_{1} \Omega_{*},
$$

where the coefficient $c_{1}$ can be computed explicitly using polar coordinates (in dimension $d=2$ ) or spherical coordinates (in dimension $d \geq 3$ ):

$$
c_{1}=\left\{\begin{array}{rr}
\int_{0}^{2 \pi} \phi(\cos \theta) \cos \theta \mathrm{d} \theta & \text { if } d=2 \\
2 \pi \int_{0}^{\pi} \phi(\cos \theta) \cos \theta \sin \theta \mathrm{d} \theta \text { if } d=3
\end{array}\right.
$$

We deduce the following result:

Proposition 2.1. The set of generalized collisional invariants associated to the unit vector $\Omega_{*}$ is given by:

$$
\mathcal{E}_{\Omega_{*}}=\left\{\psi_{\Omega_{*}}(\omega)=\alpha+u \cdot \omega, \quad \text { with } \alpha \in \mathbb{R} \text { and } u \text { orthogonal to } \Omega_{*}\right\} .
$$

Proof. Fix $\Omega_{*} \in \mathbb{S}^{d-1}$ and take $f$ satisfying (2.20). Hence, $\bar{\Omega}_{f}(2.16)$ is proportional to $\Omega_{*}$. Integrating the collisional operator $Q$ against $\omega$ leads to:

$$
\int_{\omega \in \mathbb{S}^{d-1}} Q(f) \omega \mathrm{d} \omega=\rho \int_{\omega \in \mathbb{S}^{d-1}} \phi_{\bar{\Omega}_{f}}(\omega) \omega \mathrm{d} \omega-\int_{\omega \in \mathbb{S}^{d-1}} f(\omega) \omega \mathrm{d} \omega=\rho c_{1} \bar{\Omega}_{f}-j_{f},
$$

where $j_{f}$ is the flux (2.16). The vectors $\bar{\Omega}_{f}$ and $j_{f}$ are both proportional to $\Omega_{*}$. Now let $\psi_{\Omega_{*}}(\omega)=\alpha+u \cdot \omega$ with $u$ orthogonal to $\Omega_{*}$. We have:

$$
\int_{\omega \in \mathbb{S}^{d-1}} Q(f) \psi_{\Omega_{*}} \mathrm{~d} \omega=0+u \cdot\left(\int_{\omega \in \mathbb{S}^{d-1}} Q(f) \omega \mathrm{d} \omega\right)=u \cdot\left(\rho c_{1} \bar{\Omega}_{f}-j\right)=0 .
$$

Conversely, denote $H$ the subset of $L^{2}\left(\mathbb{S}^{d-1}\right)$ of the functions $f$ satisfying the constraint (2.20). Notice that $H$ is of codimension $d-1$ in $L^{2}\left(\mathbb{S}^{d-1}\right)$ and that $Q$ is a (linear) projection operator on $H$. Therefore, the image of $Q(H)$ is of codimension $d$. Thus, the set of functions satisfying (2.19) (i.e. $\psi \in \operatorname{Im}\left(\left.Q\right|_{E}\right)^{\perp}$ ) is of dimension $d$. We deduce that the set $\mathcal{E}_{\Omega_{*}}$ includes all the GCI.

Knowing the generalized collisional invariant of $Q$, we can now investigate the limit of equation (2.13). More precisely, we can prove the following:

Theorem 2.1. Suppose the solution $f^{\varepsilon}$ of (2.13) has a limit when $\varepsilon \rightarrow 0$. Then, the asymptotic limit $f^{0}$ satisfies:

$$
f^{\varepsilon}(\mathbf{x}, \omega) \stackrel{\varepsilon \rightarrow 0}{\longrightarrow} \rho^{0}(\mathbf{x}) \phi\left(\Omega^{0}(\mathbf{x}) \cdot \omega\right),
$$


with $\rho^{0}$ and $\Omega^{0}$ satisfying the following system:

$$
\begin{aligned}
& \partial_{t} \rho+c_{1} \nabla_{\mathbf{x}} \cdot(\rho \Omega)=0 \\
& \rho\left(\partial_{t} \Omega+c_{2} \Omega \cdot \nabla_{\mathbf{x}} \Omega\right)+\lambda P_{\Omega^{\perp}} \nabla_{\mathbf{x}} \rho=0,
\end{aligned}
$$

where $P_{\Omega^{\perp}}=\mathrm{Id}-\Omega \otimes \Omega$ is the projection onto the orthogonal hyperplane to $\Omega, c_{1}$ is defined in (2.22) and:

$$
\begin{aligned}
c_{2} & = \begin{cases}\frac{1}{c_{1}} \int_{0}^{2 \pi} \phi(\cos \theta) \cos 2 \theta \mathrm{d} \theta & \text { if } d=2, \\
\frac{\pi}{c_{1}} \int_{0}^{\pi} \phi(\cos \theta)\left(2 \cos ^{2} \theta-\sin ^{2} \theta\right) \sin \theta \mathrm{d} \theta & \text { if } d=3,\end{cases} \\
\lambda & = \begin{cases}\frac{1}{c_{1}} \int_{0}^{2 \pi} \phi(\cos \theta) \sin ^{2} \theta \mathrm{d} \theta & \text { if } d=2, \\
\frac{\pi}{c_{1}} \int_{0}^{\pi} \phi(\cos \theta) \sin ^{3} \theta \mathrm{d} \theta & \text { if } d=3 .\end{cases}
\end{aligned}
$$

We leave the proof of the theorem in Appendix A.1.

\section{Discretization of the models}

In this section we discuss the discretizations techniques for the three models presented in the previous section. We develop three numerical schemes illustrated in figure 2 to discretize each model. From now on, we consider specifically a two dimensional settings. The numerical schemes can be easily generalized to three dimensional settings.
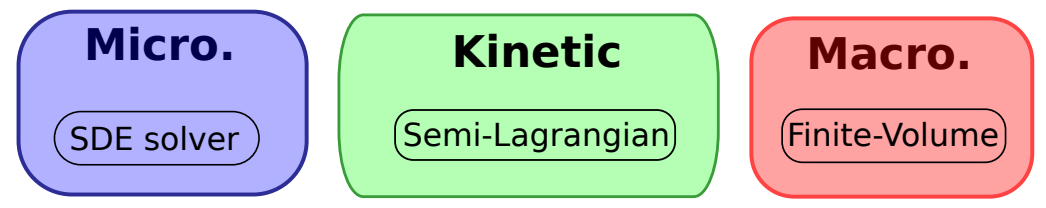

Fig. 2. Three different schemes to solve the dynamics at the three different levels (i.e., microscopic, kinetic and macroscopic levels).

\subsection{Discretization of the particle model}

Concerning the particle model described by equations $(2.1)(2.2)$, the algorithm proposed consists to approximate this continuous Markov process by a discrete one. We fix a number of $N$ agents, a time step $\Delta t$ and an interaction kernel $K(|\mathbf{x}|)$. The choices of the interaction kernel are done such that fair comparisons with the numerical schemes defined on a mesh are possible. Thus, we choose

$$
K(|\mathbf{x}|)=\left\{\begin{array}{lll}
1 & \text { if } & |\mathbf{x}| \leq R \\
0 & \text { if } & |\mathbf{x}|>R
\end{array}\right.
$$

where the radius $R$ takes the same values of the mesh sizes used for the numerical discretizations of the kinetic and hydrodynamic models. Starting from an initial state $\mathbf{x}_{k}^{0}$ and $\omega_{k}^{0}$, the algorithm consists in the following steps 
- Push particles, i.e. $\mathbf{x}_{k}^{n+1}=\mathbf{x}_{k}^{n}+\Delta t \omega_{k}^{n}$ (we take $c=1$ ).

- For each particle, compute the number of particles in its neighborhood by using the expression of the collision kernel $K(|\mathbf{x}|)$ defined in (3.1).

- Compute the average direction $\Omega_{k}$ using (2.4).

- With probability $\exp (-\nu \Delta t)$ the particle does not change direction, i.e. $\omega_{k}^{n+1}=\omega_{k}^{n}$.

- With probability $1-\exp (-\nu \Delta t)$ the particle changes direction and its new direction is sampled from the distribution $\phi\left(\Omega_{k} \cdot \omega_{k}\right)$, i.e. $\omega_{k}^{n+1}=\omega_{k}^{*}$.

To compare the results of this numerical method with the kinetic and macroscopic dynamics, the collision frequency $\nu$ is taken as $1 / \varepsilon$. When $\varepsilon$ is small, we expect to have the particle simulation getting closer to the kinetic dynamics. In addition, simulations are performed several times and the results are averaged in terms of density and mean direction. The details of this averaging is given in the numerical test section.

The comparisons between models will be performed using macroscopic quantities (i.e. density $\rho$ and average direction $\mathbf{u}$ ), thus one needs to estimate density and average orientation from the particles. Here, many different methods can be employed, our choice is to represent the macroscopic quantities on the same grid used for the numerical discretization of the kinetic and macroscopic equations. More precisely, macroscopic quantities are obtained by direct summation of the masses and momentum of the particles in each cell.

\subsection{Discretization of the kinetic model}

Concerning the discretization of the kinetic model which we recall here (the superscript $\varepsilon$ is omitted in the sequel)

$$
\partial_{t} f^{\varepsilon}(\mathbf{x}, \omega, t)+\omega \cdot \nabla_{\mathbf{x}} f^{\varepsilon}(\mathbf{x}, \omega, t)=\frac{1}{\varepsilon}\left(\rho(\mathbf{x}, t) \phi_{\bar{\Omega}_{f}^{\varepsilon}}(\omega)-f^{\varepsilon}(\mathbf{x}, \omega, t)\right),
$$

we consider a semi-Lagrangian technique. This is a deterministic numerical method which permits to have noiseless numerical simulations for the mesoscopic model. Another possibility would be to use a Monte-Carlo method to discretize the kinetic equation. We have decided to use the semi-Lagrangian method since the results can be compare efficiently with the macroscopic model (2.25)-(2.26). The general idea of the semi-Lagrangian method used is to fix a grid in the velocity space and to transform the kinetic equation in a set of linear hyperbolic equations with source terms. We refer to [21] for the detailed description of this numerical method, here we recall only the basic principles.

Let introduce a Cartesian grid $\mathcal{V}$ of $\mathbb{R}^{2}$ in the two dimensional velocity space. This grid is such that

$$
\mathcal{V}=\left\{\mathbf{v}_{k}=k \Delta v-\mathbf{v}_{0}, k=0, . ., N-1\right\} .
$$

where $\mathbf{v}_{0}=[-1,-1]$ which means we discretize the square $[-1,1] \times[-1,1]$. From the above definition the direction $\omega_{k}$ is defined as the angle between the component 


$\begin{array}{llllll}\text { January } & 28, & 2016 & 12: 15 & \text { WSPC/INSTRUCTION } & \text { FILE }\end{array}$

of discrete velocity $\mathbf{v}_{k}$ in the $x$-direction $\mathbf{v}_{k, \mathbf{x}}$ and in the $y$ direction $\mathbf{v}_{k, y}$. In this setting, the continuous distribution function $f$ is replaced by a $N$-vector where each component is assumed to be an approximation of the distribution function $f$ at location $\omega_{k}$, i.e. $f_{k}(\mathbf{x}, t) \approx f\left(\mathbf{x}, \omega_{k}, t\right)$. The fluid quantities are then obtained from $f_{k}$ thanks to discrete summations on $\mathcal{V}$ :

$$
\rho(\mathbf{x}, t)=\sum_{k} f_{k}(\mathbf{x}, t) \Delta v, \quad \Omega(\mathbf{x}, t)=\frac{j(\mathbf{x}, t)}{|j(\mathbf{x}, t)|}, \quad j(\mathbf{x}, t)=\sum_{k} \omega_{k} f_{k}(\mathbf{x}, t) .
$$

Thanks to the above discrete velocity approximation we get that the original kinetic equation (3.2) is replaced by a set of $N$ evolution equations for $f_{k}$ of the form

$$
\partial_{t} f_{k}+\omega_{k} \cdot \nabla_{\mathbf{x}} f_{k}=\frac{1}{\varepsilon}\left(\mathcal{E}_{k}-f_{k}\right),
$$

where $\mathcal{E}_{k}$ is a suitable approximation of $\rho(\mathbf{x}, t) \phi_{\bar{\Omega}_{f}}(\omega)$, i.e. $\mathcal{E}_{k} \approx \rho(\mathbf{x}, t) \phi_{\bar{\Omega}_{f}}\left(\omega_{k}\right)$. We now use a first order time splitting between the transport and the collisional operators for each equation (3.4). The transport part (left-hand side) is exactly solved (i.e. without using a spatial mesh), whereas the collsional part (right hand side) is solved on the velocity grid.

Let $f_{j, k}^{0}=f\left(\mathbf{x}_{j}, \omega_{k}, t=0\right)$ be the pointwise initial data on the $\mathbf{x}_{j}$ mesh points of the spatial grid. Let also $\mathcal{E}_{j, k}^{0}=\rho\left(\mathbf{x}_{j}, t=0\right) \phi_{\bar{\Omega}_{f}\left(\mathbf{x}_{j}, t=0\right)}\left(\omega_{k}\right)$ be the initial distribution to which particles relax over time. We describe the first step of the method $\left[t^{0} \rightarrow t^{1}\right]$ starting at $t^{0}=0$, we then generalize to an arbitrary time step. Due to the splitting the first stage is reduced to the solution of $N$ linear transport equations of the form:

$$
\partial_{t} f_{k}+\omega_{k} \cdot \nabla_{\mathbf{x}} f_{k}=0, \quad k=0, \ldots, N-1 .
$$

In order to solve this part, we define for each of the $N$ equations a piecewise constant function in the two dimensional space as

$$
\bar{f}_{k}\left(\mathbf{x}, t^{0}=0\right)=f_{j, k}^{0} \quad \forall \mathbf{x} \in\left[\mathbf{x}_{j-1 / 2}, \mathbf{x}_{j+1 / 2}[, \quad k=0, \ldots, N-1 .\right.
$$

which means that they are constant over a square of dimension corresponding to the length of the mesh. Thanks to this reconstruction, the exact solution of the $N$ equations at time $t^{1}=t^{0}+\Delta t=\Delta t$ is simply given by

$$
\bar{f}_{k}^{*}(\mathbf{x})=\bar{f}\left(\mathbf{x}-\omega_{k} \Delta t\right), \quad k=0, \ldots, N-1 .
$$

Now, to complete one time step, we need to compute the solution of the interaction part of the equation. First, We solve the right hand side of (3.4) on the grid points

$$
\partial_{t} f_{j, k}=\frac{1}{\varepsilon}\left(\mathcal{E}_{j, k}-f_{j, k}\right), \quad k=0, \ldots, N-1,
$$

for each point $\mathbf{x}_{j}$ of the grid. Initial data are given by the result of the transport step in the points $\mathbf{x}_{j}$ at time $t^{1}=t^{0}+\Delta t: \bar{f}_{k}^{*}\left(\mathbf{x}_{j}\right), \quad k=0, \ldots, N-1$. To solve (3.6), it remains to define the value of the equilibrium distribution $\mathcal{E}$ at the center of the cell after the transport. We need for that to estimate the values of the density and the average direction at the cell centers. To do so, we sum the local value of the 
discrete distribution $f$ at location $\mathbf{x}_{j}$ over the velocity set and multiplied by the generalized discrete collision invariants:

$$
\sum_{k} f_{j, k}^{*} \Delta v=\rho_{j}^{*} \quad \text { and } \quad P_{\bar{\Omega}_{f}^{\perp}}\left(\sum_{k} f_{j, k}^{*} \omega_{k} \Delta v\right)=\Omega_{j}^{*},
$$

where $f_{j, k}^{*}=\bar{f}_{k}^{*}\left(\mathbf{x}_{j}\right)$. This is enough to define the distribution $\rho(\mathbf{x}, t) \phi_{\bar{\Omega}_{f}}(\omega)$ at time $t^{1}$ in the cell centers, i.e. $\mathcal{E}_{j, k}^{1}$. Finally, the solution of the relaxation step becomes

$$
f_{j, k}^{1}=\exp (-\Delta t / \varepsilon) \bar{f}_{j, k}^{*}+(1-\exp (-\Delta t / \varepsilon)) \mathcal{E}_{j, k}^{1} .
$$

Now, the new values of the distribution $f$ at time $t^{1}=t^{0}+\Delta t=\Delta t$ at locations $\mathbf{x}_{j}$ are known. However, in order to proceed to the next time step, the distribution $f$ has to be known everywhere. One choice consists in considering that the new distribution of orientation $\rho\left(\mathbf{x}, t^{1}\right) \phi_{\bar{\Omega}_{f}\left(\mathbf{x}, t^{1}\right)}(\omega)$ has the same form as the distribution $f$ in space for each $\omega_{k}$. Starting from the pointwise value of $\mathcal{E}$ a piecewise constant function in space $\overline{\mathcal{E}}_{k}$ for each orientation $\omega_{k}$ is defined in the following way

$$
\overline{\mathcal{E}}_{k}^{*}(\mathbf{x})=\overline{\mathcal{E}}_{k}\left(\mathbf{x}, t^{1}\right)=\mathcal{E}_{j, k}^{1}, \quad \forall \mathbf{x} \text { s.t. } \bar{f}_{k}^{*}(\mathbf{x})=\bar{f}_{k}^{*}\left(\mathbf{x}_{j}\right) .
$$

This ends one step of the numerical scheme. Given now the value of the distribution function $\bar{f}_{k}^{n}(\mathbf{x})$, for all $k=0, \ldots, N-1$, and all $\mathbf{x} \in \mathbb{R}^{2}$ at time $t^{n}$, the value of the distribution at time $t^{n+1}, \bar{f}_{k}^{n+1}(\mathbf{x})$ can be simply computed as

$$
\begin{gathered}
\bar{f}_{k}^{*}(\mathbf{x})=\bar{f}_{k}^{n}\left(\mathbf{x}-\mathbf{v}_{k} \Delta t\right), \\
\bar{f}_{k}^{n+1}(\mathbf{x})=\exp (-\Delta t / \varepsilon) \bar{f}_{k}^{*}(\mathbf{x})+(1-\exp (-\Delta t / \varepsilon)) \overline{\mathcal{E}}_{k}^{n+1}(\mathbf{x}),
\end{gathered}
$$

where $\overline{\mathcal{E}}_{k}^{n+1}(\mathbf{x})$ is a piecewise constant function with the discontinuities located in the same positions (different for each $k$ of the lattice) as the distribution $\bar{f}_{k}^{*}$. It is computed considering the moments values in the center of each spatial cell after the transport step. These moments are obtained by computing $\sum_{k} f_{j, k}^{*} \Delta v$ and $P_{\bar{\Omega}_{f}^{\perp}}\left(\sum_{k} f_{j, k}^{*} \omega_{k} \Delta v\right)$ where $f_{j, k}^{*}$ is the value that the distribution function takes after the transport in the center of each spatial cell.

This scheme is unconditionally stable, however, for accuracy reasons, the time step $\Delta t$ is chosen in order to satisfy the condition $\Delta t / \Delta x<1$ since the maximum speed of the particles is fixed to one. This also permit a fair comparison with the particle simulations since the two time steps coincide.

\subsection{Discretization of the macroscopic model}

To discretize the macroscopic equations, we use the numerical scheme developed in [34]. For completeness we summarize the scheme here. The non-conservative model (2.25)-(2.26) can be seen as the asymptotic limit of the following system:

$$
\begin{aligned}
& \partial_{t} \rho+c_{1} \nabla_{\mathbf{x}} \cdot(\rho \Omega)=0, \\
& \partial_{t}(\rho \Omega)+c_{2} \nabla_{\mathbf{x}} \cdot(\rho \Omega \otimes \Omega)+\lambda \nabla_{\mathbf{x}} \rho=\frac{\rho^{\eta}}{\eta}\left(1-|\Omega|^{2}\right) \Omega,
\end{aligned}
$$


as $\eta \rightarrow 0$. The numerical scheme consists in solving the system (3.8)-(3.9) in two steps (splitting method). First we use a finite-volume method to solve the conservative part (left-hand side):

$$
\begin{aligned}
& \partial_{t} \rho+c_{1} \nabla_{\mathbf{x}} \cdot(\rho \Omega)=0, \\
& \partial_{t}(\rho \Omega)+c_{2} \nabla_{\mathbf{x}} \cdot(\rho \Omega \otimes \Omega)+\lambda \nabla_{\mathbf{x}} \rho=0 .
\end{aligned}
$$

Then, we solve the relaxation part (right-hand side):

$$
\begin{aligned}
& \partial_{t} \rho=0, \\
& c_{1} \partial_{t}(\rho \Omega)=\frac{\rho^{\eta}}{\eta}\left(1-|\Omega|^{2}\right) \Omega .
\end{aligned}
$$

One can pass to the limit $\eta \rightarrow 0$ as the relaxation operator becomes a mere normalization of $\Omega$.

\section{Numerical investigations}

In the previous Sections, we have presented several numerical schemes to approach the three levels of descriptions of the self-alignment jump process (i.e. particle system (2.1)(2.2), kinetic equation (2.7) and macroscopic model (2.25)-(2.26)). In this section, we would like to put to the test the numerical schemes and the theory by investigating numerically different problems. As stated in the introduction, we try to introduce a systematic approach which permits to validate both the model and the numerical methods.

First, we concentrate of the kinetic equation (2.11) and its semi-Lagrangian discretization. We analyze the convergence of the scheme with respect to its space discretization. We do not perform a similar convergence study for the particle dynamics since this is a standard approach. Instead, we are more interested in performing several comparisons between the hierarchy of the models proposed (i.e. micro, kinetic, macro). In particular, we show that the particle system converges toward the kinetic equation as the number of particles $N$ get larger and that the kinetic equation converges to the macroscopic model as the jump frequency $\nu$ increases. Finally, we investigate a novel type of pattern formation observed using a vortex configuration as initial condition. The solutions, obtained by approaching the kinetic equation and the macroscopic equation, present a variety of patterns and are superimposed in time confirming that the two models give identical solutions in the limit $\nu \rightarrow \infty$. This is also confirmed by a convergence test with respect to the time and space discretization in which the relative error between the macroscopic and the kinetic discretizations is measured.

In the following, all the simulations are done in a $2 \mathrm{D}$ domain $D=[0,10] \times[0,10]$ using periodic boundary conditions.

\subsection{A Convergence test for the semi-Lagrangian scheme}

In this first part, we analyze the convergence of the semi-Lagrangian method depending on its spatial and time discretization $(\Delta x, \Delta y$ and $\Delta t)$. With this aim, we 
solve the kinetic equation (2.7) starting with the following initial condition:

$$
f_{0}(\mathbf{x}, \theta)=C \exp \left(-\left|\mathbf{x}-\mathbf{x}_{c}\right|^{2}\right) \mathbb{1}_{\left[-\frac{\pi}{2}, \frac{\pi}{2}\right]}(\theta),
$$

where $C$ is a normalization constraint, $\mathbf{x}_{c}=(5,5)$ is the center of the domain and $\mathbb{1}$ is the indicator function (see figure $3 \mathbf{a}$ ). We perform the simulation with a high resolution in velocity $\left(\Delta \theta=\frac{2 \pi}{100}\right)$ and in time discretization $\left(\Delta t=10^{-3}\right)$ as we aim to measure the accuracy of the scheme in space. In figure $3 \mathbf{b}-\mathbf{c}$, we plot the solution at $t=2,4$ time unit with $\Delta x=\Delta y=.25$ unit space. We observe that the solution both diffuses in space and moves in the $x$-direction as one can expect since the velocity distribution is centered around $\theta=0$ initially.

To estimate the accuracy of the method, we use as a benchmark $f_{*}$ the solution computed with $\Delta x=\Delta y=\frac{1}{2^{5}}=.03125$ unit space. Then, we estimate the solutions with $\Delta x=1, \frac{1}{2}, \frac{1}{2^{2}}, \frac{1}{2^{3}}$ keeping $\Delta y=\Delta x$. To evaluate the convergence, we compute the $L^{1}$ distance between the density $\rho(x, y, t)$ estimated at $t=2$ with various $\Delta x$ and $\Delta y$ :

$$
\operatorname{error}(\Delta x)=\left\|\rho_{\Delta x}(., t=2)-\rho_{*}(., t=2)\right\|_{L^{1}},
$$

where $\rho_{*}(x, y, t)$ is the mass distribution of the benchmark solution $f_{*}$ (i.e. $\left.\rho(x, y, t)=\int_{\theta} f(x, y, \theta, t) \mathrm{d} \theta\right)$. As we observe in figure $3 \mathbf{d}$, the error is decaying linearly with respect to $\Delta x$ showing that the method is first order accurate in space.

\subsection{From microscopic to macroscopic description}

We now investigate numerically the links between the three levels of descriptions of our self-alignment jump process. With this aim, we analyze in different regimes the solution of a Riemann problem taking the following initial condition

$$
\rho_{0}(x, y)=\left\{\begin{array}{l}
1, \text { if } x<5 \\
2, \text { if } x>5
\end{array} \quad, \quad \theta_{\Omega_{0}}(x, y)=\left\{\begin{array}{c}
1.5, \text { if } x<5 \\
1.83, \text { if } x>5
\end{array}\right.\right.
$$

where $\rho_{0}$ denotes the mass distribution and $\theta_{\Omega_{0}}$ the average direction. The solution is homogeneous in the $y$-direction and therefore we only plot a slice in the $x$-direction. At the particle and kinetic levels, one also needs to specify the density distribution in velocity variable $\theta$. We use for that a Von Mises distribution (2.6) with $\sigma$ given by the noise level of the dynamics $(\sigma=.1)$.

First, we compare the results given by the particle dynamics $(2.1)(2.2)$ with the solution of the kinetic equation (2.7) for $\nu=1$. In figure 4, we plot the density $\rho$ and velocity $\mathbf{u}$ in the $x$-direction for both solutions. We average the solution of the particle dynamics over 100 simulations to reduce fluctuation. With $N=$ $10^{3}$ particles, the particle simulation is more diffusive in space compared with the kinetic solution. But as the number of particles increases, the solutions get closer to the kinetic solution, and at $N=10^{5}$ the curves are almost identical. Such result illustrates the propagation of chaos: as $N \rightarrow \infty$, the particle system (2.1)(2.2) converges to the (deterministic) solution of the kinetic equation (2.7). 

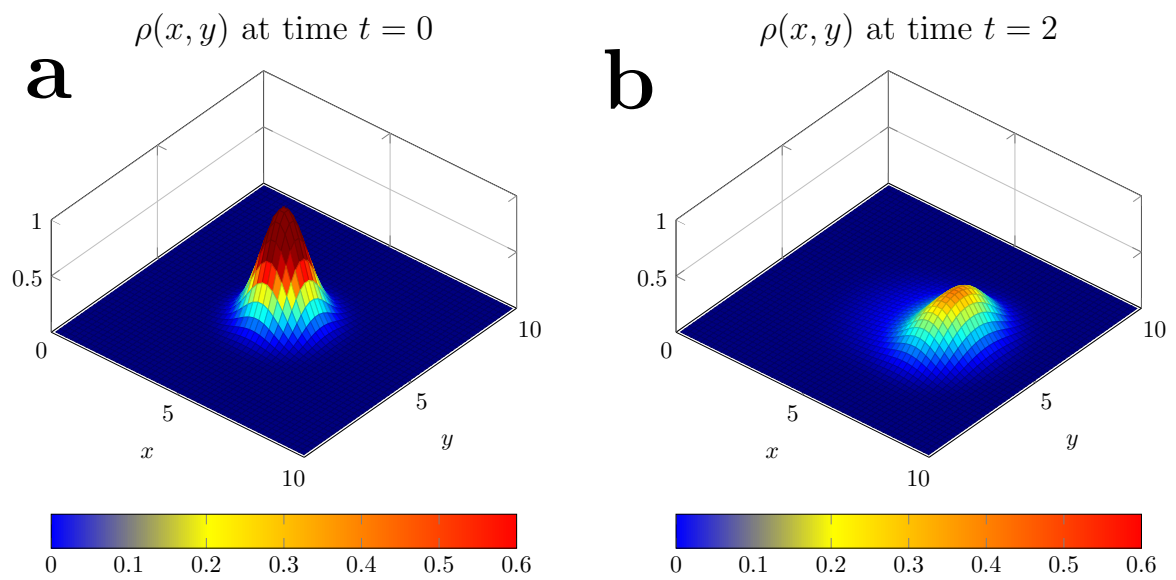

$\rho(x, y)$ at time $t=4$
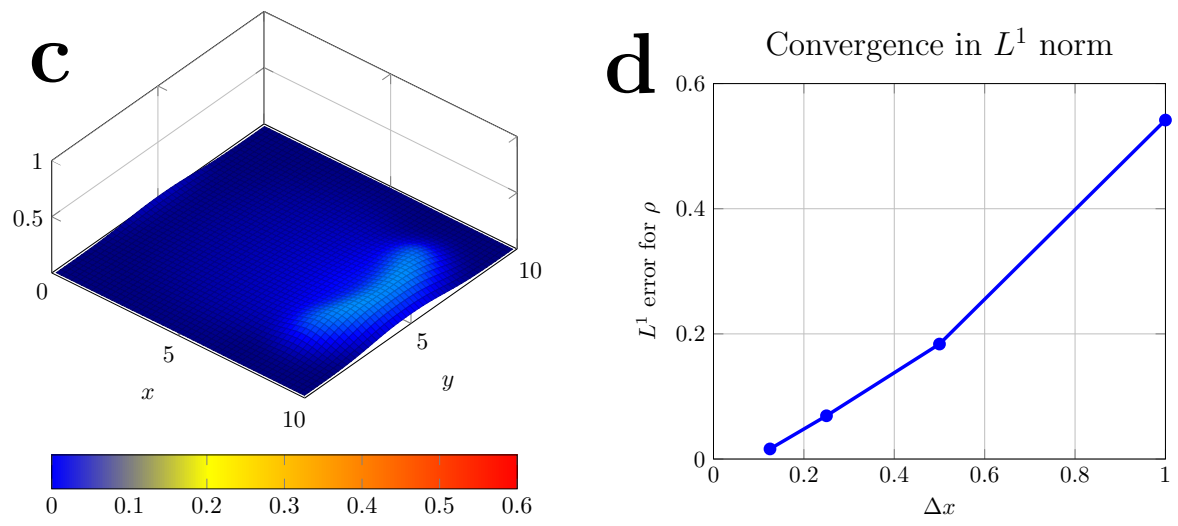

Fig. 3. a,b,c) Mass distribution $\rho$ of the solution to the kinetic equation (2.11) with the initial condition (4.1) at $t=0,2$ and 4 unit time. Parameters: $c=1, \nu=.1, \sigma=1, \phi$ uniform (2.5), $\Delta t=10^{-3}, \Delta \theta=2 \pi / 100$. d) Accuracy of the scheme in space with the error given by (4.2).

Second, we compare kinetic and macroscopic models. To link the two descriptions, one needs to increase the jump frequency $\nu$ in the kinetic model in order to be close to the equilibrium state. This corresponds to work in an hydrodynamic scaling (2.12). Thus, as the $\nu$ increases, one expects that the solution of the kinetic equation (2.7) converge to the solution of the macroscopic model (2.25)-(2.26) as it has been shown analytically in theorem 2.1. In figure 5 , we plot the density $\rho$ and average velocity $\mathbf{u}$ of the kinetic solution for different frequency $\nu$. At $\nu=1$, the kinetic solution is more diffusive compared with the macroscopic solution as 
expected. With $\nu=10$, we clearly distinguish the shock and rarefaction profiles. Still, we do not yet observe a sharp transition (discontinuity) of the shock profile at $x \approx 3.4$ space unit of the macroscopic model. But at $\nu=100$, the kinetic solution and macroscopic solution become almost identical, even near the discontinuous profile (i.e. $x \approx 3.4$ and 5.3 space unit).

\subsection{Vortex solution}

In this paragraph, we would like to investigate the behaviors of our model in a more complex setting. We consider a fully $2 \mathrm{D}$ simulation with an initial condition given by a vortex configuration (defined below). We compare the results obtained from the kinetic (2.7) and macroscopic models (2.25)-(2.26). To do so, we solve the kinetic model in a regime close to the so-called thermodynamical equilibrium taking $\nu=100$. The exact initial condition is as follows:

$$
f_{0}(\mathbf{x}, \theta)=C \exp \left(-\sin \left(\theta-\varphi_{\mathbf{x}}\right)\right)
$$

where $C$ is a normalization constraint and $\varphi_{\mathbf{x}}$ is the angle between the $x$-axis and the vector $\left(\mathbf{x}-\mathbf{x}_{c}\right)$ where $\mathbf{x}_{c}=(5,5)$ is the center of the domain. In figure $6 \mathbf{a}-\mathbf{f}$, we plot the spatial density $\rho(x, y)$ (color) and average velocity $\mathbf{u}$ (arrows) at different time interval ( $t=5,10,15$ unit times) solving the kinetic equation (figure 6-left) and macroscopic model (figure 6-right). The symmetry of the initial condition is preserved through the simulation and the profiles of the solutions keep alternating between circle and square shape presenting novel patterns. As the system is non conservative, there is no guarantee that the solution will eventually dissipate and stabilize to a stationary state ${ }^{24}$. The absence of entropy makes the time asymptotic of the solution challenging to analyze. The change of model comes with a cost: solving the macroscopic model took 12.4 seconds whereas 107.9 seconds was required to solve the kinetic model.

Notice that the solution of the macroscopic solution (figure 6-right) is more diffusive. The kinetic simulation presents more sharp transition. This is due to the different level of precision of the two numerical schemes employed. This is made more clear by a convergence analysis of the same kind of the one performed for the case of the sole scheme for the kinetic equation. In more details, we measure the the $L_{1}$ norm of the error between the densities computed by two schemes when the final time $t=1$ reducing the space and time steps. Thus we measure

$$
\operatorname{error}(t=1)=\left\|\rho_{\text {macro }}(t=1)-\rho_{\text {kinetic }}(t=1)\right\|_{L^{1}} .
$$

for $\Delta x=\frac{2}{5}, \frac{1}{5}, \frac{1}{10}$ keeping $\Delta y=\Delta x$ and diminishing the time step $\Delta t$ accordingly by using the CFL condition defined in the previous Section. In figure 7 , this error is reported which shows that the two schemes and thus the two models converge once the mesh becomes smaller. In other words, the two models describe the same type of dynamics. 

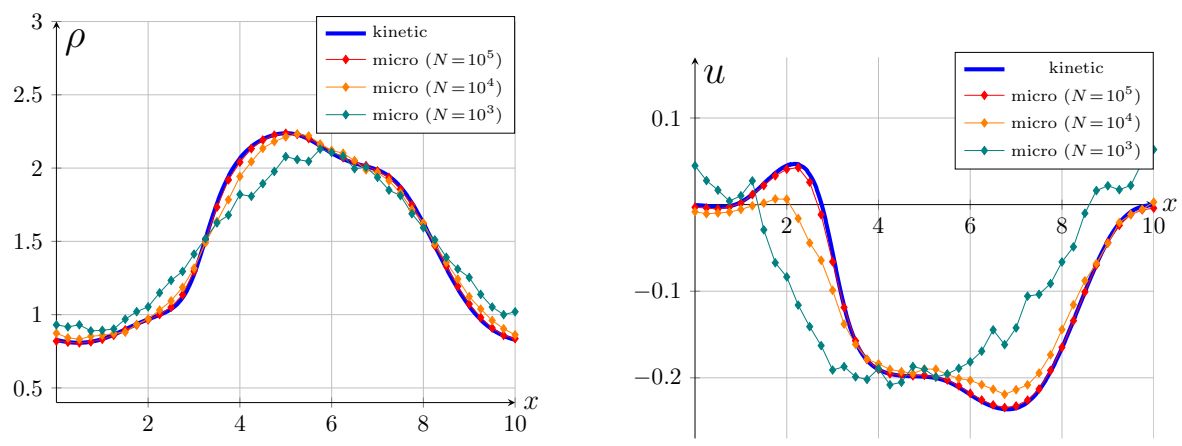

Fig. 4. Solution of the Riemann problem (4.3) at $t=4$ time units for the particle system (diamond marks) and kinetic equation (line). Left: Density distribution $\rho$. Right: average velocity $u$ in the $x$-direction. As the number of particles $N$ increases, the solutions to the particle system converge to the kinetic solution. Parameters particle simulation: $c=1, \nu=1, \sigma=.1, \phi$ Von Mises (2.6), $\Delta t=10^{-2}, R=10^{-1}$. Additional parameters for the kinetic simulation: $\Delta x=\Delta y=10^{-2}$, $\Delta \theta=2 \pi / 100$.
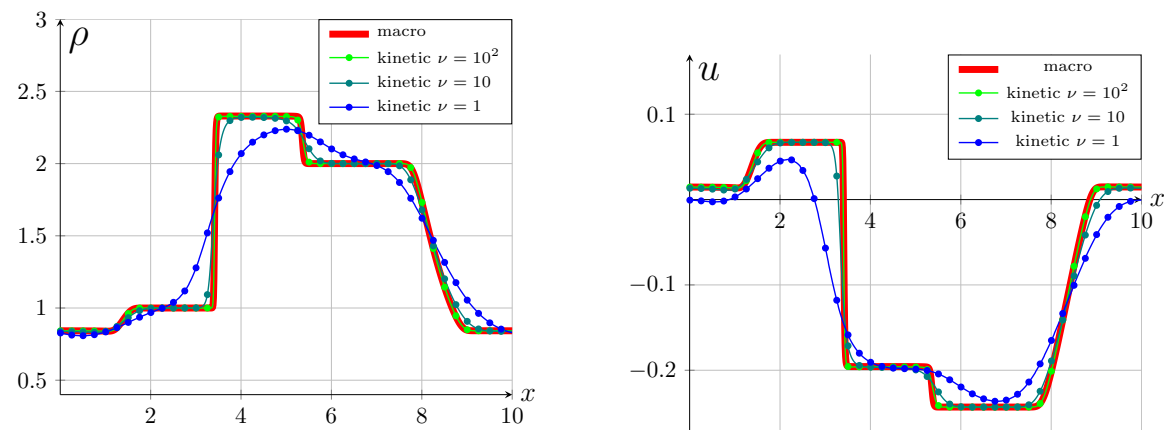

Fig. 5. Solution of the Riemann problem (4.3) at $T=4$ time units for the kinetic equation (circle) and macroscopic model (line). Left: Density distribution $\rho$. Right: average velocity $u$ in the $x$ direction. The solutions to the kinetic equation converge to the corresponding macroscopic model as the frequency of jumps $\nu$ increases. Parameters kinetic simulations: see figure 4. Parameter macroscopic model: $c_{1}=.949, c_{2}=.854, \lambda=.1, \Delta x=10^{-2}, \Delta t=10^{-2}$.

\section{Conclusions}

In this paper, we have derived an hydrodynamic model from a system of selfpropelled particles which align through a jump process. The method has consisted in first introducing a kinetic description of the model used obtained in the limit of infinitely many particles. Then, in a regime close to thermodynamic equilibrium, we have derived a macroscopic description. A discretization strategy has been proposed 
January 28

28

$2016 \quad 12: 15$

WSPC/INSTRUCTION

FILE

Vicsek'BGK'm3as'160127
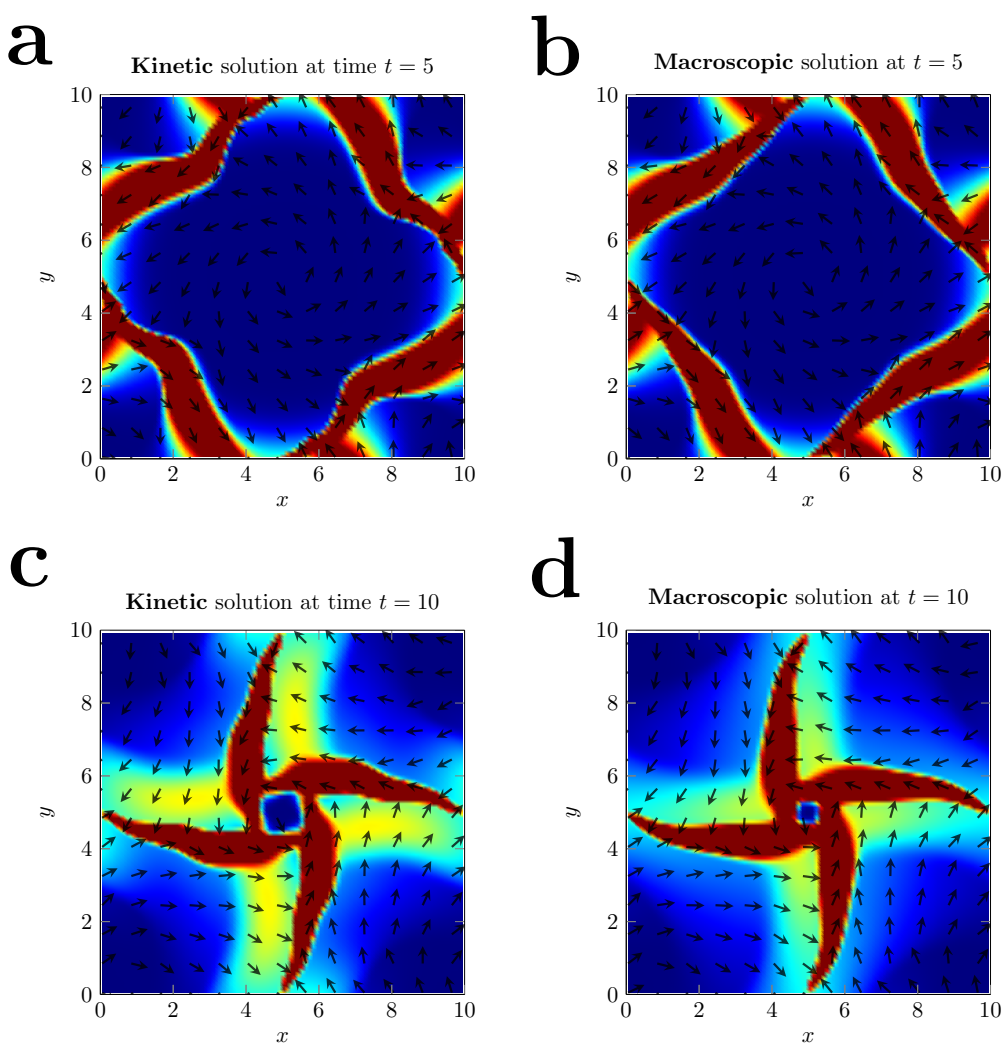

e

f

Kinetic solution at time $t=15$
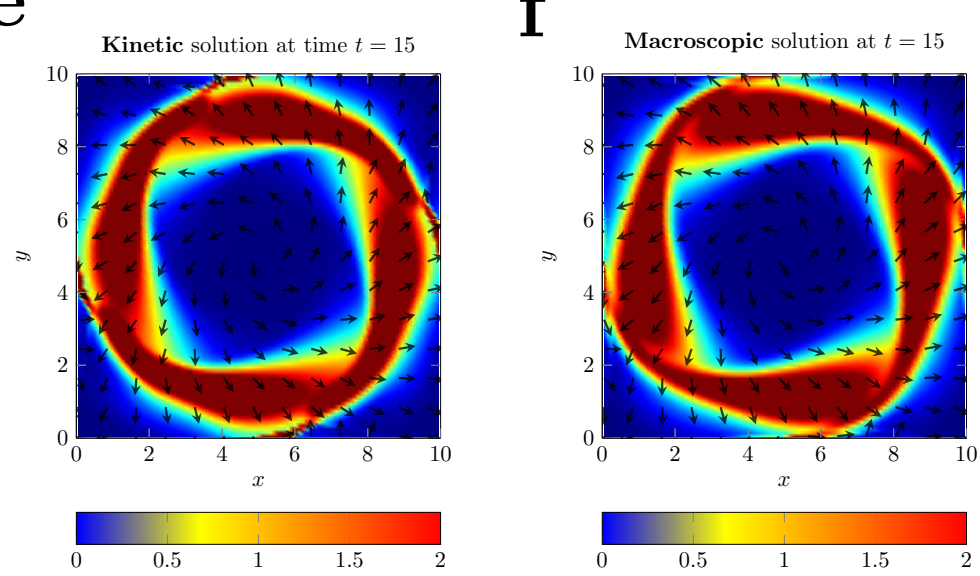

Fig. 6. Solutions of the kinetic (left) and macroscopic (right) equation for the initial condition (4.4). The solutions keep alternating between circular and straight shapes. Due to the absence of entropy, there is no guarantee that the solutions will converge toward a stationary state. Parameters: $\Delta t=5 \cdot 10^{-2}, \Delta x=\Delta y=10^{-1}$. Kinetic equation: $c=1, \nu=100, \sigma=.1, \phi$ Von Mises (2.6), $\Delta \theta=2 \pi / 100$. 


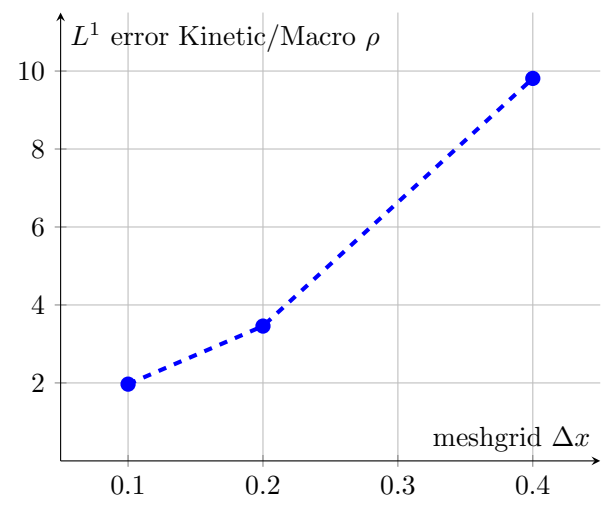

Fig. 7. $L^{1}$ error between the spatial density $\rho$ of the kinetic and macroscopic model (4.5). The error is decreasing with the reduction of the mesh size and time step.

for each of the three systems. Then, we studied the numerical convergence of the schemes and compared their behaviors going from the finer description (particle systems) to the macroscopic limit. This analysis showed that the different descriptions give not only comparable but equivalent solutions in suitable regimes. Therefore, in those regimes, kinetic or macroscopic descriptions are a better choice compare with the particle description. They provide more accurate solutions in shorter time and thus provide more reliable results which might be impossible to obtain from particle simulations. As a perspective of this work, we would like to expand the numerical validation strategy proposed in this paper to other active particles models and extend the jump process model to multispecies agents in order to consider particles with different characteristics acting in the same environment.

\section{Appendix A. Appendices}

\section{A.1. Proof Theorem 2.1}

Multiplying the equation (2.13) by $\varepsilon$ and passing to the limit $\varepsilon \rightarrow 0$, we deduce that $Q\left(f^{0}\right)=0$. Thus,

$$
f^{0}(x, \omega)=\rho^{0}(x) \phi\left(\Omega^{0} \cdot \omega\right) .
$$

To obtain the equations satisfied by $\rho^{0}$ and $\Omega^{0}$, we use the collisional invariants. First, using $\psi=1$, we integrate (2.13) in $\omega$ and deduce the equation of mass conservation:

$$
\partial_{t} \rho^{\varepsilon}+\nabla_{x}\left(j^{\varepsilon}\right)=0
$$

where the flux $j^{\varepsilon}$ is defined in (2.14). At the limit $\varepsilon \rightarrow 0$, combining (A.1) and (2.21), we deduce the equation of mass conservation (2.25). 
In a second step, we use the generalized collisional invariant. Let $v^{\varepsilon} \in \mathbb{R}^{d}$ be a vector orthogonal to $\Omega^{\varepsilon}$. We integrate (2.13) against $\omega \cdot v^{\varepsilon}$ :

$$
\left(\partial_{t} \rho^{\varepsilon} u^{\varepsilon}+\int_{\omega \in \mathbb{S}^{d-1}} \omega \cdot \nabla_{x} f^{\varepsilon} \omega \mathrm{d} \omega\right) \cdot v^{\varepsilon}=O(\varepsilon) .
$$

At the limit $\varepsilon \rightarrow 0$, we obtain:

$$
\left(\partial_{t} c_{1} \rho^{0} \Omega^{0}+\int_{\omega \in \mathbb{S}^{d-1}} \omega \cdot \nabla_{x}\left(\rho^{0} \phi\left(\Omega^{0} \cdot \omega\right)\right) \omega \mathrm{d} \omega\right) \cdot v^{0}=0,
$$

with $c_{1}$ given by $(2.22)$. In other words, noting $P_{\left(\Omega^{0}\right) \perp}$ the projection onto the orthogonal hyperplane of $\Omega^{0}$, we have:

$$
P_{\left(\Omega^{0}\right)^{\perp}}\left(\partial_{t} c_{1} \rho^{0} \Omega^{0}+\int_{\omega \in \mathbb{S}^{d-1}} \omega \cdot \nabla_{x}\left(\rho^{0} \phi\left(\Omega^{0} \cdot \omega\right)\right) \omega \mathrm{d} \omega\right)=0 .
$$

In the following, we drop the super-script for clarity. Thus, we write:

$$
\begin{aligned}
P_{\Omega^{\perp}}\left(c_{1} \partial_{t}(\rho \Omega)+\nabla_{x} \cdot\left(\rho \int_{\omega \in \mathbb{S}^{d-1}} \phi(\Omega \cdot \omega) \omega \otimes \omega \mathrm{d} \omega\right)\right) & =0 \\
P_{\Omega^{\perp}}(A+B) & =0 .
\end{aligned}
$$

Expanding the expression of $A$, we deduce that:

$$
P_{\Omega^{\perp}} A=c_{1} P_{\Omega^{\perp}}\left(\partial_{t} \rho \Omega+\rho \partial_{t} \Omega\right)=c_{1} \rho \partial_{t} \Omega,
$$

since $\partial_{t} \Omega$ is orthogonal to $\Omega$ (i.e. $\left\langle\partial_{t} \Omega, \Omega\right\rangle=\frac{1}{2} \partial_{t}|\Omega|^{2}=0$ ).

To simplify the expression of $B$, we use the following lemma:

Lemma Appendix A.1. Let $\Omega \in \mathbb{S}^{d-1}$ with $d=2$ or $d=3$. Then

$$
\int_{\omega \in \mathbb{S}^{d-1}} \phi(\Omega \cdot \omega) \omega \otimes \omega \mathrm{d} \omega=\alpha \Omega \otimes \Omega+\beta \mathrm{Id},
$$

where the coefficients $c_{2}$ and $\lambda$ are given (resp.) by (2.27) and (2.28).

$$
\begin{aligned}
& \alpha=\left\{\begin{array}{cc}
\int_{0}^{2 \pi} \phi(\cos \theta) \cos 2 \theta \mathrm{d} \theta & \text { if } d=2, \\
\pi \int_{0}^{\pi} \phi(\cos \theta)\left(2 \cos ^{2} \theta-\sin ^{2} \theta\right) \sin \theta \mathrm{d} \theta & \text { if } d=3,
\end{array}\right. \\
& \beta= \begin{cases}\int_{0}^{2 \pi} \phi(\cos \theta) \sin ^{2} \theta \mathrm{d} \theta & \text { if } d=2, \\
\pi \int_{0}^{\pi} \phi(\cos \theta) \sin ^{3} \theta \mathrm{d} \theta & \text { if } d=3 .\end{cases}
\end{aligned}
$$

Using this lemma, we can expand the expression of $B$ (A.2):

$$
\begin{aligned}
P_{\Omega^{\perp}} B & =P_{\Omega^{\perp}}\left(\nabla_{x} \cdot(\alpha \rho \Omega \otimes \Omega+\beta \rho \mathrm{Id})\right) \\
& =\alpha P_{\Omega^{\perp}}\left(\Omega \otimes \Omega \nabla_{x} \rho+\rho \Omega \cdot \nabla_{x} \Omega+\rho\left(\nabla_{x} \cdot \Omega\right) \Omega\right)+\beta P_{\Omega^{\perp}} \nabla_{x} \rho .
\end{aligned}
$$

As $|\Omega|=1$, we deduce that $\Omega \cdot \nabla_{x} \Omega$ is orthogonal to $\Omega$ since $\left\langle\Omega \cdot \nabla_{x} \Omega, \Omega\right\rangle=\Omega$. $\nabla_{x}|\Omega|^{2}=0$. Thus, $P_{\Omega^{\perp}}\left(\Omega \cdot \nabla_{x} \Omega\right)=\Omega \cdot \nabla_{x} \Omega$. Combined with $P_{\Omega^{\perp}}(\Omega)=0$, we finally obtain:

$$
P_{\Omega^{\perp}} B=\alpha \rho \Omega \cdot \nabla_{x} \Omega+\beta P_{\Omega^{\perp}} \nabla_{x} \rho .
$$


Combining (A.3) and (A.7), we deduce (2.26).

Proof. (Lemma Appendix A.1)

- We start with the case of dimension $d=2$. Using polar coordinates $\omega=\cos \theta \Omega+$ $\sin \theta \Omega^{\perp}$ with $\Omega^{\perp}$ an orthonormal vector to $\Omega$, we obtain:

$$
\begin{aligned}
\int_{\omega \in \mathbb{S}^{1}} \phi(\Omega \cdot \omega) \omega \otimes \omega \mathrm{d} \omega & =\int_{0}^{2 \pi} \phi(\cos \theta)\left[\begin{array}{cc}
\cos ^{2} \theta & \cos \theta \sin \theta \\
\cos \theta \sin \theta & \sin ^{2} \theta
\end{array}\right] \mathrm{d} \theta \\
& =\int_{0}^{2 \pi} \phi(\cos \theta)\left[\begin{array}{cc}
\cos ^{2} \theta & 0 \\
0 & \sin ^{2} \theta
\end{array}\right] \mathrm{d} \theta
\end{aligned}
$$

by symmetry. Thus,

$$
\int_{\omega \in \mathbb{S}^{d-1}} \phi(\Omega \cdot \omega) \omega \otimes \omega \mathrm{d} \omega=\gamma \Omega \otimes \Omega+\beta \Omega^{\perp} \otimes \Omega^{\perp}
$$

with $\gamma=\int_{0}^{2 \pi} \phi(\cos \theta) \cos ^{2} \theta \mathrm{d} \theta$ and $\beta$ given by (A.6). Using that $\Omega^{\perp} \otimes \Omega^{\perp}=\mathrm{Id}-$ $\Omega \otimes \Omega$, we finally obtain (A.4) where:

$$
\alpha=\gamma-\beta=\int_{0}^{2 \pi} \phi(\cos \theta)\left(\cos ^{2} \theta-\sin ^{2} \theta\right) \mathrm{d} \theta
$$

which leads to the expression (A.5) of $\alpha$.

- In the case $d=3$, we use spherical coordinates: let $u$ and $v$ such that $\{u, v, \Omega\}$ is an orthonormal basis of $\mathbb{R}^{3}$. Then,

$$
\begin{aligned}
\int_{\omega \in \mathbb{S}^{2}} \phi(\Omega \cdot \omega) \omega \otimes \omega \mathrm{d} \omega & =\int_{\theta=0}^{\pi} \phi(\cos \theta) \sin \theta \int_{\varphi=0}^{2 \pi}\left(\begin{array}{c}
\cos \varphi \sin \theta \\
\sin \varphi \sin \theta \\
\cos \theta
\end{array}\right) \otimes\left(\begin{array}{c}
\cos \varphi \sin \theta \\
\sin \varphi \sin \theta \\
\cos \theta
\end{array}\right) \mathrm{d} \varphi \mathrm{d} \theta \\
& =\int_{\theta=0}^{\pi} \phi(\cos \theta) \sin \theta\left[\begin{array}{ccc}
\pi \sin ^{2} \theta & 0 & 0 \\
0 & \pi \sin ^{2} \theta & 0 \\
0 & 0 & 2 \pi \cos ^{2} \theta
\end{array}\right] \mathrm{d} \theta \\
& =\beta(\mathrm{Id}-\Omega \otimes \Omega)+\gamma \Omega \otimes \Omega,
\end{aligned}
$$

with $\beta$ given by (A.6) and $\gamma=2 \pi \int_{\theta=0}^{\pi} \phi(\cos \theta) \sin \theta \cos ^{2} \theta \mathrm{d} \theta$. We deduce the expression (A.4) with $\alpha=\gamma-\beta$ leading to (A.5).

\section{A.2. Coefficients of the macroscopic model}

We estimate numerically the coefficients $c_{1}, c_{2}, \lambda$ of the macroscopic models (2.25)(2.26) depending on the level of noise. The noise is encoded in either $\eta$ or $\sigma$ depending of the choise of distribution $\phi$ (see (2.5) and (2.6)). In figure 8, we estimate the coefficients in dimension $d=2$. We observe that the transport coefficients $c_{1}, c_{2}$ decay as the noise increases, whereas the pressure coefficient $\lambda$ increases. 
Coefficients 2D, $\phi$ uniform

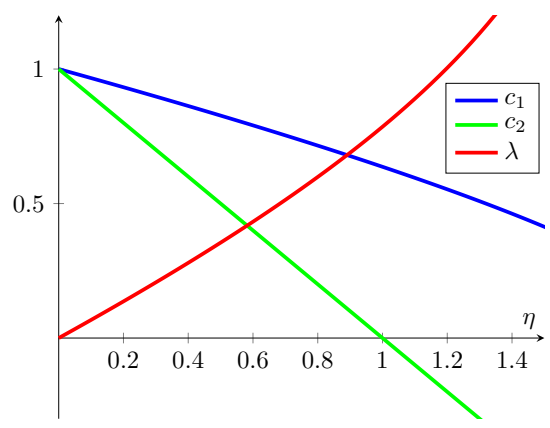

Coefficients 2D, $\phi$ Von Mises

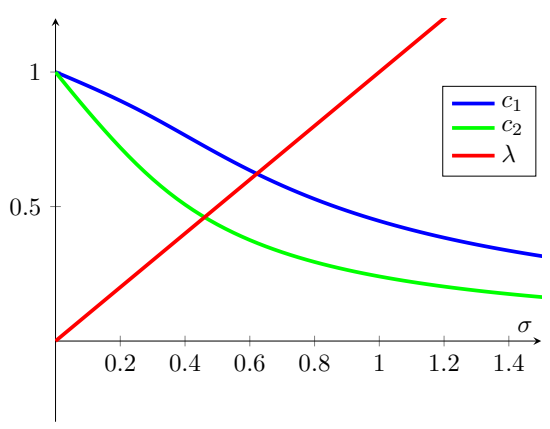

Fig. 8. Estimation of the macroscopic coefficients in dimension $d=2$. Left: $\phi$ uniform (2.5). Right: $\phi$ Von Mises (2.6). Parameters for the estimation of the integrals: $\Delta \theta=2 \pi \cdot 10^{-7}$.

\section{Acknowledgment}

This work has been supported by the French 'Agence Nationale pour la Recherche (ANR)' in the frame of the contract 'MOTIMO' (ANR-11-MONU-009-01) and by the NSF grants DMS 1515592 and RNMS11-07444 (KI-Net). The authors would like to thanks Professor Pierre Degond for stimulating discussions.

\section{References}

1. G. Albi and L. Pareschi. Binary interaction algorithms for the simulation of flocking and swarming dynamics. Multiscale Modeling \& Simulation, 11(1):1-29, 2013.

2. M. Aldana and C. Huepe. Phase Transitions in Self-Driven Many-Particle Systems and Related Non-Equilibrium Models: A Network Approach. Journal of Statistical Physics, 112(1):135-153, 2003.

3. I. Aoki. A simulation study on the schooling mechanism in fish. Bulletin of the Japanese Society of Scientific Fisheries (Japan), 1982.

4. A. Baskaran and C. Marchetti. Hydrodynamics of self-propelled hard rods. Physical Review E, 77(1):011920, 2008.

5. N. Bellomo, A. Bellouquid, Y. Tao, and M. Winkler. Towards a Mathematical Theory of Keller-Segel Models of Pattern Formation in Biological Tissues. Mathematical Models and Methods in Applied Sciences, 2015.

6. N. Bellomo and F. Brezzi. Complexity and Multiscale Features of Large Systems of Self-propelled Particles. Mathematical Models and Methods in Applied Sciences, To appear, 2015.

7. N. Bellomo and J. Soler. On the mathematical theory of the dynamics of swarms viewed as complex systems. Mathematical Models and Methods in Applied Sciences, 22(supp01):1140006, 2012.

8. E. Bertin, M. Droz, and G. Grégoire. Hydrodynamic equations for self-propelled particles: microscopic derivation and stability analysis. Journal of Physics A: Mathematical and Theoretical, 42(44):445001, 2009.

9. J. A Carrillo, M. Fornasier, J. Rosado, and G. Toscani. Asymptotic Flocking Dynamics 
for the kinetic Cucker-Smale model. SIAM J. Math. Anal., 42:218-236, 2010.

10. H. Chaté, F. Ginelli, G. Grégoire, and F. Raynaud. Collective motion of self-propelled particles interacting without cohesion. Physical Review E, 77(4):46113, 2008.

11. Y. Chuang, M. R D'Orsogna, D. Marthaler, A. L Bertozzi, and L. S Chayes. State transitions and the continuum limit for a $2 \mathrm{~d}$ interacting, self-propelled particle system. Physica D: Nonlinear Phenomena, 232(1):33-47, 2007.

12. I. D Couzin, J. Krause, R. James, G. D Ruxton, and N. R Franks. Collective Memory and Spatial Sorting in Animal Groups. Journal of Theoretical Biology, 218(1):1-11, 2002.

13. F. Cucker and S. Smale. Emergent Behavior in Flocks. IEEE Transactions on automatic control, 52(5):852, 2007.

14. P. Degond, G. Dimarco, and T. Mac. Hydrodynamics of the Kuramoto-Vicsek model of rotating self-propelled particles. Mathematical Models and Methods in Applied Sciences, 24(02):277-325, 2014

15. P. Degond, G. Dimarco, T. Mac, and N. Wang. Macroscopic models of collective motion with repulsion. Communications in Mathematical Sciences, 13(6):1615-1638, 2015.

16. P. Degond, A. Frouvelle, and J-G. Liu. Macroscopic limits and phase transition in a system of self-propelled particles. Journal of nonlinear science, 23(3):427-456, 2013.

17. P. Degond, A. Frouvelle, J-G. Liu, S. Motsch, and L. Navoret. Macroscopic models of collective motion and self-organization. Séminaire Laurent Schwartz - EDP et applications, 2013.

18. P. Degond and J. Hua. Self-Organized Hydrodynamics with congestion and path formation in crowds. Journal of Computational Physics, 237:299-319, 2013.

19. P. Degond and S. Motsch. Continuum limit of self-driven particles with orientation interaction. Mathematical Models and Methods in Applied Sciences, 18(1):1193-1215, 2008.

20. P. Degond and S. Motsch. Large Scale Dynamics of the Persistent Turning Walker Model of Fish Behavior. Journal of Statistical Physics, 131(6):989-1021, 2008.

21. G. Dimarco and R. Loubere. Towards an ultra efficient kinetic scheme. Part I: Basics on the BGK equation. Journal of Computational Physics, 255:680-698, December 2013.

22. R. Durrett. Probability: theory and examples. Cambridge university press, 2010.

23. F. Filbet, P. Laurençot, and B. Perthame. Derivation of hyperbolic models for chemosensitive movement. Journal of Mathematical Biology, 50(2):189-207, 2005.

24. F. Filbet and T. Rey. A rescaling velocity method for dissipative kinetic equations. Applications to granular media. Journal of Computational Physics, 248:177-199, September 2013.

25. M. Fornasier, J. Haskovec, and G. Toscani. Fluid dynamic description of flocking via the Povzner-Boltzmann equation. Physica D: Nonlinear Phenomena, 240(1):21-31, 2011.

26. A. Frouvelle and J-G. Liu. Dynamics in a kinetic model of oriented particles with phase transition. SIAM Journal on Mathematical Analysis, 44(2):791-826, 2012.

27. G. Grégoire and H. Chaté. Onset of Collective and Cohesive Motion. Physical Review Letters, 92(2):25702, 2004.

28. S. Y Ha and J. G Liu. A simple proof of the Cucker-Smale flocking dynamics and mean-field limit. Communications in Mathematical Sciences, 7(2):297-325, 2009.

29. M. Hauray and S. Mischler. On Kac's chaos and related problems. Journal of Functional Analysis, 266(10):6055-6157, 2014.

30. S. Henkes, Y. Fily, and C. Marchetti. Active jamming: Self-propelled soft particles at 
high density. Physical Review E, 84(4):040301, 2011.

31. Pierre-Emmanuel Jabin. A review of the mean field limits for Vlasov equations. Kinetic and Related Models, 7(4):661-711, November 2014.

32. D. Koch and G. Subramanian. Collective hydrodynamics of swimming microorganisms: Living fluids. Annual Review of Fluid Mechanics, 43:637-659, 2011.

33. A. Mogilner, L. Edelstein-Keshet, L. Bent, and A. Spiros. Mutual interactions, potentials, and individual distance in a social aggregation. Journal of Mathematical Biology, 47(4):353-389, 2003.

34. S. Motsch and L. Navoret. Numerical simulations of a non-conservative hyperbolic system with geometric constraints describing swarming behavior. Multiscale Modeling Simulation, 9(3):1253-1275, 2011.

35. S. Motsch and E. Tadmor. A New Model for Self-organized Dynamics and Its Flocking Behavior. Journal of Statistical Physics, 144(5):923-947, August 2011.

36. L. Pareschi and G. Toscani. Interacting multiagent systems: kinetic equations and Monte Carlo methods. Oxford University Press, 2013.

37. J. K Parrish, S. V Viscido, and D. Grunbaum. Self-organized fish schools: an examination of emergent properties. Biological Bulletin, Marine Biological Laboratory, Woods Hole, 202(3):296-305, 2002.

38. V. I. Ratushnaya, D. Bedeaux, V. L. Kulinskii, and A. V. Zvelindovsky. Collective behavior of self-propelling particles with kinematic constraints: The relation between the discrete and the continuous description. Physica A: Statistical Mechanics and its Applications, 381:39-46, 2007.

39. A. S Sznitman. Topics in propagation of chaos. école d'été de probabilités de SaintFlour XIX-1989. Lecture Notes in Math, 1464:165-251, 1989.

40. John Toner, Yuhai Tu, and Sriram Ramaswamy. Hydrodynamics and phases of flocks. Annals of Physics, 318(1):170-244, 2005.

41. Y. Tu, J. Toner, and M. Ulm. Sound waves and the absence of Galilean invariance in flocks. Physical review letters, 80(21):4819, 1998.

42. T. Vicsek, A. Czirók, E. Ben-Jacob, I. Cohen, and O. Shochet. Novel type of phase transition in a system of self-driven particles. Physical Review Letters, 75(6):12261229, 1995.

43. T. Vicsek and A. Zafeiris. Collective motion. Physics Reports, 517(3):71-140, 2012. 